\title{
Tendencias temáticas e investigativas en las facultades de educación en Colombia: una mirada a las revistas científicas
}

\author{
Researching and Thematic Tendencies on Colombia's \\ Faculties of Education: a View on the Journals
}

Carlos Rincón Zabala*

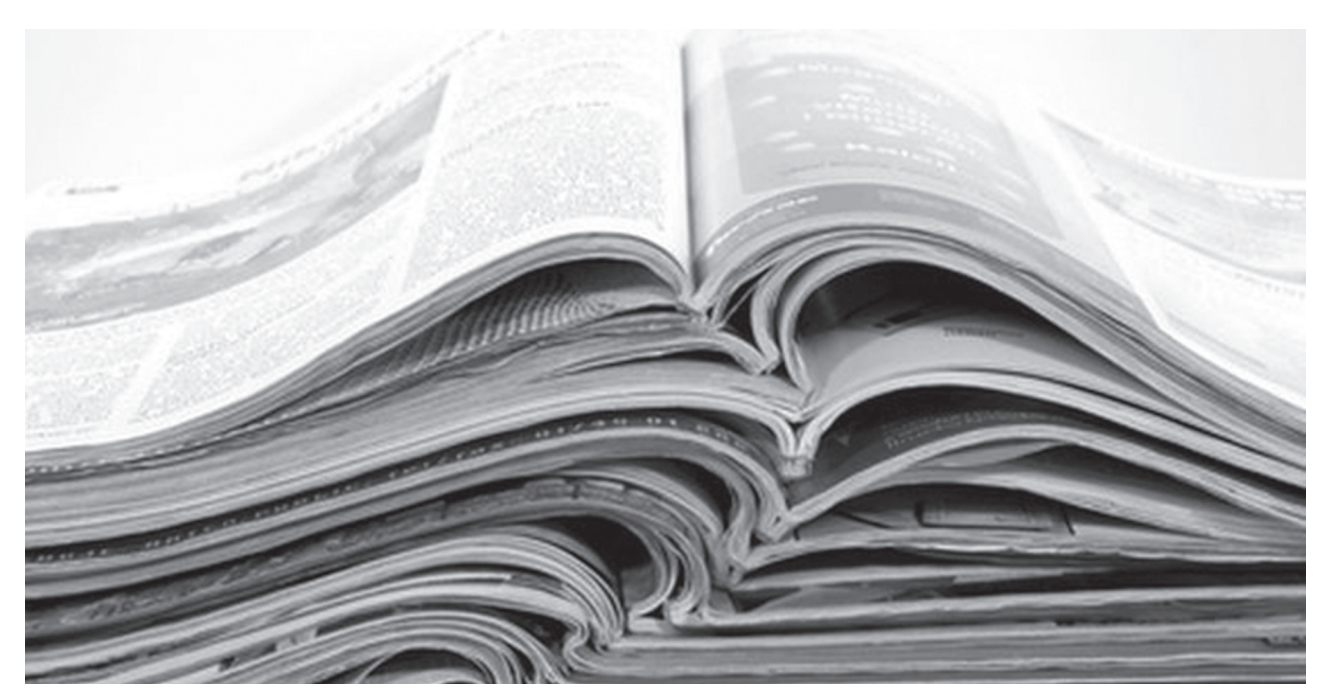

\section{Resumen}

En el siguiente artículo se realiza una indagación respecto a la concepción de la investigación que ha sido adoptada en las facultades de educación de varias universidades colombianas. Para ello, se propone una revisión de carácter virtual-digital, una mirada rigurosa de los últimos cinco índices de las principales revistas científicas en los que la educación y la pedagogía se hayan convertido en las protagonistas. Basado en referentes, estadísticas, escalafones nacionales e internacionales, el texto no premia una búsqueda por la definición del concepto mismo de investigación, sino que pretende rastrear, realizar un examen sobre las tendencias investigativas que desde la academia - y particularmente desde los departamentos de educación- permiten conocer cómo esta (la investigación) ha sido pensada.

Citar este artículo como: Rincón Zabala, C. (2016). Tendencias temáticas e investigativas en las facultades de educación en Colombia: una mirada a las revistas científicas. Revista Papeles, 8(15), pp. 10-20.

Fecha de recibido: enero 7 de 2016.

Fecha de aceptación: junio 15 de 2016.

* Licenciado en Lengua Castellana e Inglés de la Universidad Antonio Nariño. Correo electrónico: karlosrincon17@gmail.com 
Palabras clave: facultades de educación, investigación, revistas científicas, tendencias investigativas.

\begin{abstract}
It is sought with this article to make an inquiry on the concept of research adopted by the departments of education in some Colombian universities. In order to achieve so, it is proposed an online-digital literary review on some of the most important journal indexes in where education and pedagogy have become in the main characters. Based on some statistics, national and international rankings as references, the paper does not pretend to inquire into what research is, that is to say, the text is not in the pursuit of defining the concept itself; instead of that, it aims to do an assessment about the researching tendencies that from the academy - especially from education departments - allow to understand how is this (the research) being thought.
\end{abstract}

Keywords: education departments, journals, research, researching tendencies.

\title{
Introducción
}

Uno de los tópicos a los que más aludió Martha Nussbaum en su discurso de aceptación del doctorado honoris causa entregado por la Universidad de Antioquia fue la crisis por la que está atravesando la educación a nivel mundial. "Cambios radicales" dentro de los sistemas políticos y económicos son, para la filósofa estadounidense, las principales causas de que estén desapareciendo de las escuelas, entre otros, la enseñanza de ciertas disciplinas que se incluyen tanto en las humanidades como en las ciencias sociales (Nussbaum, 2010 y 2015). La paulatina disminución de estas áreas dentro de los currículos educativos compromete directamente a disciplinas como la pedagogía, por ejemplo; al mismo tiempo que exige, por parte de las facultades y departamentos de las universidades, nuevas dinámicas que promuevan adecuarse - $\mathrm{o}$ en su defecto, hacerse a un lado- a las necesidades que el sistema educativo vaya requiriendo. Tal necesidad se va haciendo cada vez más evidente y se materializa, por ejemplo, en la manera en que las mismas instituciones educativas dirigen sus procesos de investigación. De esta manera, ciertas disciplinas pertenecientes a la gran área de ciencias sociales, o "ciencias blandas", entre las que se incluyen las ciencias de la educación (Colciencias, 2016), comienzan a verse relegadas y a ocupar un lugar secundario en las preferencias investigativas dentro de cada departamento e institución; lo cual se manifiesta en los productos que estas presentan al público académico: artículos científicos y estructura de las revistas de divulgación científica, sobre todo.

Adicional a lo ya mencionado, y como lo manifestaron en su momento Quintero y Ruiz (2004), la investigación en educación en Colombia ha estado permeada por una constante incertidumbre e inconstancia: investigar en educación requiere de una articulación de distintas necesidades e intereses que permiten la convivencia de elementos de carácter social, económico y, por supuesto, educativo; los cuales no han podido, de acuerdo con los autores, gozar de una adecuada fusión (Quintero y Ruiz, 2004). Es precisamente la falta de articulación entre estos elementos lo que genera el poco impacto y la descontextualización de la investigación educativa. Además de esto, el profesor Santiago González (2011) enumera otra serie de dificultades que ha presentado la investigación (no solo en el campo educativo) en el país:

- Falta de formación y de capacitación de los docentes en el campo investigativo. 
- Saturación de asignaturas en los planes de estudio, las cuales no dejan espacio suficiente a los procesos formativos de investigación.

- Transmisión de conocimientos desactualizados, los cuales no generan desarrollo de formas que puedan producir nuevos conocimientos pedagógicos y/o didácticos.

- Las líneas de investigación no se encuentran suficientemente articuladas a las problemáticas contextuales en las cuales se inscribe el saber pedagógico sobre el cual versa la investigación (González, 2011).

Del mismo modo, y sumado a lo ya expuesto, la propuesta del mismo Ministerio de Educación Nacional (MEN) pone de manifiesto otra arista a las dificultades de la investigación en educación. En los nuevos Lineamientos de Calidad para las Licenciaturas en Educación, presentados hace dos años (2014), el MEN no le asigna una relevancia adecuada a lo que ellos mismos denominan procesos de investigación. En aquel texto, el MEN hace mención a la necesidad de desarrollar en los docentes distintos mecanismos que les permitan desempeñarse satisfactoriamente en el campo investigativo (MEN, 2014); sin embargo, poco se dice sobre el modo en que ello debe llevarse a cabo; y menos aún, acerca de los criterios que se han de tener en cuenta para poder pensar la investigación de un modo distinto al hasta ahora hecho: donde se asume, y con ferviente razón, que la investigación en

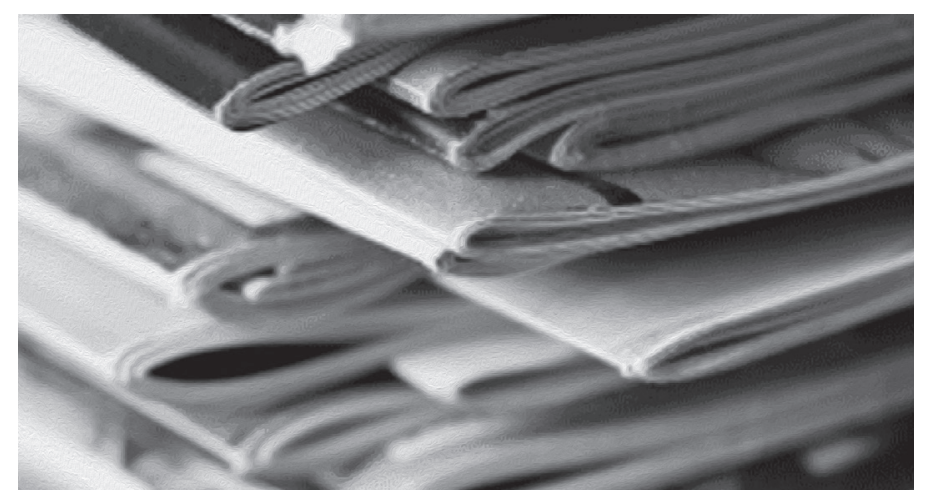

educación ha estado estructurada de acuerdo con criterios no muy bien delimitados; tal y como lo demuestran, por ejemplo, los trabajos de Lobato (2010), González (2011) y Quintero y Ruiz (2004). La investigación, así entendida, se considera como otro de los tantos requisitos (no más importante que el resto) con los que deben cumplir las instituciones encargadas de formar docentes.

Lo hasta aquí mencionado podría sintetizarse, de un modo general, de la siguiente manera: hay una crisis en la educación mundial (Nussbaum, 2010), una crisis que se pone de manifiesto en las universidades, de las universidades pasa a las facultades y departamentos (incluyendo las de educación), la problemática se filtra en los procesos de investigación y, finalmente, se evidencia en los productos: las revistas científicas.

Debido a esta serie de cuestiones expresadas en los resultados de la investigación docente en Colombia, hacemos la pregunta por la orientación, los ejes, las tendencias temáticas e investigativas sobre las cuales está girando la investigación en educación, desde sus respectivas facultades y departamentos. Por consiguiente, la pregunta orientadora de este artículo será: ¿cómo se está entendiendo la investigación en las facultades de educación colombianas y cómo ello se evidencia en las últimas cinco publicaciones de las principales revistas especializadas en el tema?

A través de una mirada a estas revistas se podrá dejar en claro -o por lo menos teorizar-qué tipo de preferencias están primando en los docentes investigadores, conforme a los temas de investigación; así como las necesidades investigativas que dentro de las mismas instituciones han sido generadas. Los trabajos realizados en el mismo campo por Quintero y Ruiz (2004) y Murcia y Guacaneme (2011) -a nivel nacional-y por Couture (2001) y Gu \& Blackmore (2016) - a nivel internacional- permitirán un mejor acercamiento; al mismo tiempo que funcionarán como puntos 
de partida que permitan entrever una parte del conjunto amplio y dinámico de relaciones en el que se encuentra envuelta la investigación en educación en Colombia; para, de esta manera, poder proponer nuevas conjeturas, realizar nuevas especulaciones sobre el carácter de la investigación educativa en las universidades del país.

\section{Propuesta metodológica}

El proceso metodológico que se llevó a cabo fue dividido en tres etapas. En la primera de ellas se buscaba determinar las revistas que deberían ser tenidas en cuenta para el rastreo e identificación de las tendencias temáticas en investigación educativa en Colombia. Para tal cometido, se realizó una búsqueda en portales de difusión científica, bibliotecas digitales y rankings académicos (nacionales e internacionales), los cuales tuvieran como propósito clasificar, de un modo general, revistas, disciplinas, materias, etc., de acuerdo con áreas de estudio. De esta manera, en el ámbito internacional, se seleccionaron el Scimago Journal Rank y se tuvieron en cuenta (a nivel latinoamericano) el portal de producción científica Dialnet, la biblioteca digital SciELO y el sistema de información académica Latindex. A nivel colombiano, el referente lo configuró el sistema de indexación Publindex, administrado por Colciencias.

Las bases, bibliotecas y rankings internacionales no proporcionaron fuentes significativas con las cuales se pudiera realizar un trabajo de revisión de revistas científicas: en el ranking de Scimago, en su última entrega, únicamente se incluyen dos revistas colombianas de educación (Scimago, 2014). Por su parte, los portales y/o bibliotecas Dialnet, SciELO y Latindex, si bien configuran una fuente rica de referencias, no presentan una clasificación sistemática de las revistas adscritas. Debido a estos inconvenientes, se tomó como fuente primaria el sistema de información e indexación académica Publindex. No obstante, gran parte de la información encontrada en los demás portales sirvieron como puntos de apoyo y de referencia - fuentes secundarias- para varios apartados de este texto.
La segunda etapa de la metodología consistió en realizar una búsqueda de todas las revistas colombianas especializadas en educación que se encontrarán clasificadas por Publindex, la fuente primaria.

El último escalafón presentado por Colciencias, en el segundo semestre de 2014, cuenta con un total de 373 revistas indexadas; de las cuales 24 se encuentran clasificadas en la categoría A1 (categoría superior), 73 en la categoría A2, 67 en la categoría B y 209 en la categoría C (Colciencias, 2015).

Para poder comprender el oriente por el cual se ha encaminado la investigación en las facultades de investigación en Colombia y, por consiguiente, poder tener una muestra más significativa para esta investigación, se tornó necesario seleccionar aquellas revistas que se encontraran clasificadas en las categorías más altas; esto es, que satisfagan de un modo más eficiente los criterios y estándares de calidad exigidos para investigaciones de esta índole (Hernández, Fernández y Baptista, 2010). Las revistas que con más convicción cumplían con estas condiciones fueron aquellas que pertenecían a las categorías A1 y A2, según los criterios de Colciencias. Desafortunadamente, en estas dos categorías solo son incluidas 7 revistas que sean especializadas en educación. Realizar un estudio con un número tan reducido de muestras, difícilmente podrá cumplir con los criterios mínimos de formalidad (y de veracidad) requeridos (Hernández, Fernández y Baptista, 2010); además, se aleja en gran medida del propósito de esta investigación. Debido a ello, para la investigación se optó por tomar como muestras de análisis todas las revistas especializadas en educación que 
Tabla 1. Revistas colombianas de educación pertenecientes a las categorías A1, A2 y B de Colciencias"

\begin{tabular}{llcc}
\hline No. & Nombre de la revista & $\begin{array}{c}\text { Institución a la que se } \\
\text { encuentra asociada } \\
\text { (siglas) }\end{array}$ & $\begin{array}{c}\text { Categoría } \\
\text { según } \\
\text { Publindex }\end{array}$ \\
\hline 1 & Profile & UNAL & A1 \\
\hline 2 & Colombian Applied Linguistics Journal & UD & A2 \\
\hline 3 & Educación y educadores & UNISABANA & A2 \\
\hline 4 & Praxis y saber & UPTC & A2 \\
\hline 5 & Revista colombiana de educación & UPN & A2 \\
\hline 6 & Revista Historia de la Educación Latinoamericana & UPTC & A2 \\
\hline 7 & Sophia: educación & UGC & A2 \\
\hline 8 & Aletheia & CINDE & B \\
\hline 9 & Educación física y deporte & UdeA & B \\
\hline 10 & GIST. Education and Learning Research Journal & UNICA & B \\
\hline 11 & HOW & ASOCOPI & B \\
\hline 12 & Revista Latinoamericana de Estudios Educativos & UdeC & B \\
\hline 13 & Lúdica Pedagógica & UPN & B \\
\hline 14 & Magis. Revista Internacional de Investigación en educación & PUJ & B \\
\hline 15 & Nodos y nudos & UPN & B \\
\hline 16 & Pedagogía y saberes & UPN & B \\
\hline 17 & Zona Próxima & Uninorte & B \\
\hline 18 & Journal of Science Education & Varios & No aplica \\
\hline
\end{tabular}

Fuente: elaboración propia

fueran incluidas tanto en las categorías A1 y A2 como en la categoría B de Colciencias.

En la indagación acerca de las revistas pertenecientes a estas tres categorías (un total de 164), se pudo constatar que únicamente 17 de ellas son especializadas en educación y/o pedagogía: el mayor número de revistas (un total de 10) es aquel que corresponde a la categoría B de Colciencias; 6 se ubican en la categoría A2, y solamente una en la categoría A1 (Colciencias, 2015). Por otra parte, y basado en la información suministrada por

* La tabla 1 presenta información parcial respecto de lo investigado sobre las revistas. Una segunda tabla fue realizada, la cual incluye más información sobre las revistas: el año de iniciación (de acuerdo con Latindex) y el año de indexación a Publindex, la ciudad sede, si se encuentra adscrita o no a una facultad de educación, etc. (Mirar Anexo 1). Varios de estos datos serán retomados en las conclusiones. varias de las fuentes secundarias (sobre todo Scimago y Latindex), se añadió a la lista una nueva revista ${ }^{1}$. En la tabla 1 se sintetiza el proceso anteriormente mencionado.

Una vez identificadas las principales revistas colombianas especializadas en educación, el tercer, y último paso de la metodología, consistió en determinar qué debería ser a analizado en las revistas. Para ello, se tuvieron en cuenta: uno, el número total de índices de cada revista que serían seleccionados para conformar la

1 La revista en cuestión es el Journal of Science Education, editada por varias instituciones universitarias del país. Si bien no hace parte del último escalafón publicado por Colciencias, se encuentra, sin embargo, registrada en la plataforma de Publindex. Además, y más relevante aún, es una de las dos revistas, junto con Magis de la PUJ, que se encuentra en el escalafón presentado por Scimago en su última publicación del año 2014 en la categoría “Educación” (Scimago, 2014). 
muestra; y dos, los criterios que permitirían clasificar las tendencias temáticas que fueran identificadas. Respecto de lo primero, se decidió revisar los últimos 5 índices de cada revista. La decisión se tomó luego de descubrir que no se evidencia concordancia en los periodos de publicación entre las distintas revistas; es decir que unas revistas publican, por ejemplo, tres números por año; mientras que otras revistas reportan una sola publicación anual. Así, si se hubiera decidido tomar como variable un periodo de tiempo particular (por ejemplo, los años 2014 y 2015), el resultado habría estado inclinado hacia aquellas revistas que publican con mayor periodicidad. De este modo, se tuvieron en cuenta para el análisis las últimas 5 publicaciones de cada revista, omitiendo el año en que fueron publicadas. Esto dio como resultado un total de 89 revistas.

Acerca de la segunda cuestión, es decir, los criterios que deberían ser tenidos en cuenta para determinar las tendencias temáticas, se realizó un proceso de selección en el que se tuvieron en cuenta tres factores: el primero, basado en las temáticas, materias, áreas y/o campos de investigación señaladas por Ospina y Murcia (2012), Murcia y Guacaneme (2011) y Murcia, Murcia y Urbina (2011). El segundo, basado en las "formas de operar la investigación" señaladas por Quintero y Ruíz (2004). Como tercer factor, se propusieron nuevos ejes de estudio, distintos a los propuestos por los anteriores autores, y teniendo en cuenta distintas tendencias temáticas que se presentaban en los índices. Es decir, aquellas tendencias que no podían ser encasilladas ni en las propuestas del primer criterio ni en las del segundo.

Como resultado de lo anterior, como criterios de clasificación para los 89 índices de revistas fueron propuestas las siguientes 7 tendencias temáticas y/o investigativas: aprendizaje; pedagogía y desarrollo; prácticas pedagógicas; ética y ciudadanía; teorías de la salud; educación en TIC; y evaluación. Es necesario aclarar que el concepto "tendencia temática" no corresponde a un área o materia particular dentro del ámbito educativo-investigativo; debido a ello, es posible que varios índices de revistas - que fácilmente podrían ser clasificados en enseñanza de la matemática o didáctica de la lengua, por ejemplo-, se vean incluidos en una misma tendencia temática, como aprendizaje, ética y desarrollo, etc.

Por otro lado, y debido a la variedad de propuestas investigativas inmersas en los índices de las revistas analizadas, cada una de las anteriores tendencias temáticas tuvo que subdividirse. Estas subdivisiones fueron denominadas subcategorías temáticas. Así, la tendencia temática aprendizaje, tuvo que subdividirse en las siguientes subcategorías: enseñanza de las ciencias; estrategias didácticas; y desarrollo cognitivo. La tendencia pedagogía y desarrollo fue dividida en las subcategorías comunicación y lenguaje; afectividad y desarrollo humano; y concepto de niñez. Prácticas pedagógicas estuvo subdividida en actos pedagógicos; educación universitaria; práctica docente; e investigación en educación. La tendencia temática ética y pedagogía la configuran las subcategorías razonamiento moral; socialización política; ciudadanía e interculturalidad; e inclusión y permanencia escolar. Teorías de la salud se encuentra subdividida en fisioterapia y promoción de la salud; y desarrollo cultural y ocio. En la tendencia educación en TIC se encuentra una sola subdivisión: TIC como mediadoras en el aprendizaje. Finalmente, la tendencia temática evaluación se ve integrada por la subcategoría evaluación y calidad.

Cada uno de los índices de cada revista fue clasificado en una única tendencia temática. Si bien es claro que no todos los números de las revistas están enfocados en una materia o asunto particular, es decir que los trabajos que las integran desarrollan investigaciones alrededor de distintos campo educativos, sí se pueden entrever los principales enfoques de cada revista, así como las relaciones que estos guardan entre ellos; cuestión que permite clasificar cada revista en una sola de las tendencias temáticas propuestas. 


\section{Resultados}

La clasificación de los índices de las revistas, de acuerdo con las tendencias temáticas propuestas, arrojó los siguientes resultados: de los 89 índices analizados, un $28 \%$, es decir, 25 índices, corresponden a la tendencia temática denominada prácticas pedagógicas. Con un 26\% y con 23 índices aparece la tendencia ética y ciudadanía. A esta le siguen las tendencias (empatadas en porcentaje y en número de índices) aprendizaje y pedagogía $y$ desarrollo con un $17 \%$ y 15 números cada una. Con porcentajes más bajos se inscriben las tendencias temáticas teorías de la salud física y educación en TIC, con un $8 \%$ y 7 números la primera, y con $3 \%$ y 3 números la segunda. Cierra la lista la tendencia evaluación, que cuenta con un solo índice y que equivale al $1 \%$ del total de índices. En la gráfica 1 se ejemplifica lo anterior:

Gráfica 1. Porcentaje de las tendencias temáticas

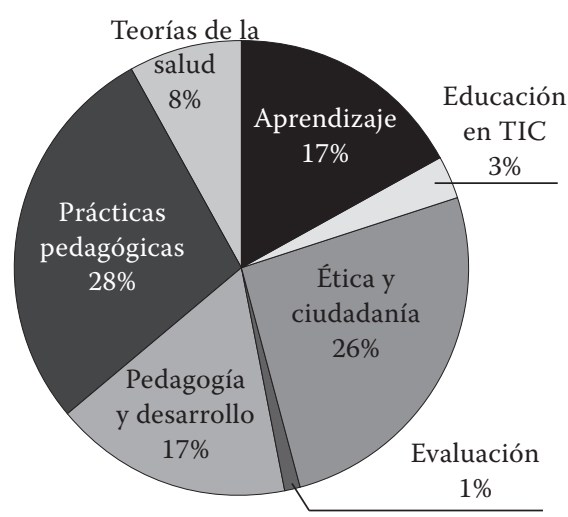

Fuente: elaboración propia

Como se propuso en la metodología, cada una de las tendencias temáticas fue subdividida en categorías particulares (subcategorías) dentro del eje temático en que fue clasificada. En las siguientes gráficas se presenta el modo en que cada tendencia temática se encuentra subdividida en sus respectivas categorías, así como el porcentaje y número de índices de revistas que fueron clasificadas en cada una.
En la tendencia prácticas pedagógicas, cada categoría temática se distribuye del siguiente modo: actos pedagógicos, 12 números (48\%); práctica docente, 6 números (24\%); educación universitaria, 4 números (16\%); e investigación en educación, 3 números (12\%). La gráfica 2 muestra la distribución en porcentajes:

Gráfica 2. Prácticas pedagógicas

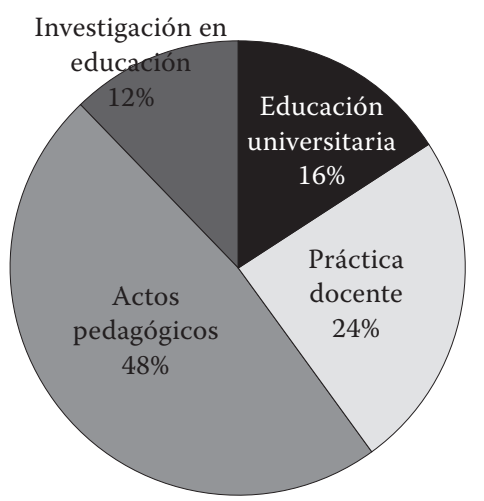

Fuente: elaboración propia

La tendencia ética y ciudadanía se encuentra subdividida, en sus respectivas categorías temáticas, como se explica a continuación: ciudadanía e interculturalidad cuenta con 11 números (48\%), socialización política con 6 (26\%), inclusión y permanencia escolar con 5 (22\%), y razonamiento moral con 1 (4\%). La gráfica 3 muestra los porcentajes:

Gráfica 3. Ética y ciudadanía

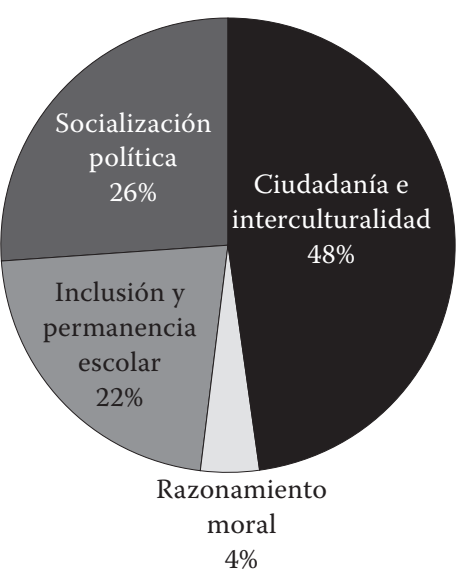

Fuente: elaboración propia 
En la tendencia aprendizaje fueron clasificados un total de 15 índices; y distribuidos en las subcategorías del siguiente modo: enseñanza de las ciencias, 10 números (67\%); estrategias didácticas, 4 números (27\%); y desarrollo cognitivo, 1 número (6\%). En la gráfica 4 se muestra la distribución en porcentajes:

Gráfica 4. Aprendizaje

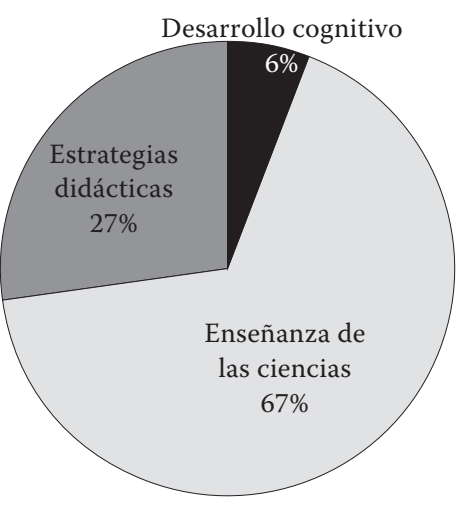

Fuente: elaboración propia

En la tendencia temática pedagogía y desarrollo, al igual que en la anterior, fueron clasificados un total de 15 números; donde la categoría temática comunicación y lenguaje cuenta con 13 de ellos (86\%), afectividad y desarrollo humano cuenta con 1 (6\%), y concepto de niñez también cuenta con 1 (6\%). La distribución en porcentajes es expresada a continuación:

\section{Gráfica 5. Pedagogía y desarrollo}

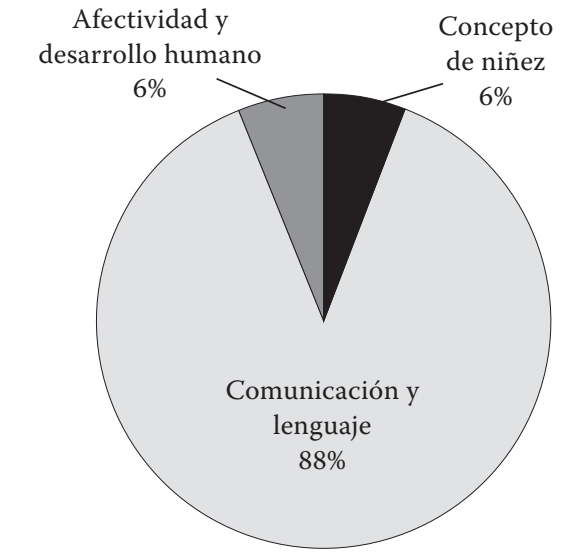

Fuente: elaboración propia
La tendencia temática teorías de la salud estuvo subdividida del siguiente modo: la subcategoría desarrollo corporal y ocio contó con 5 números (71\%), mientras que fisioterapia y promoción de la salud contó con 2 números (29\%). Esto se muestra en la gráfica 6:

Gráfico 6. Teorías de salud

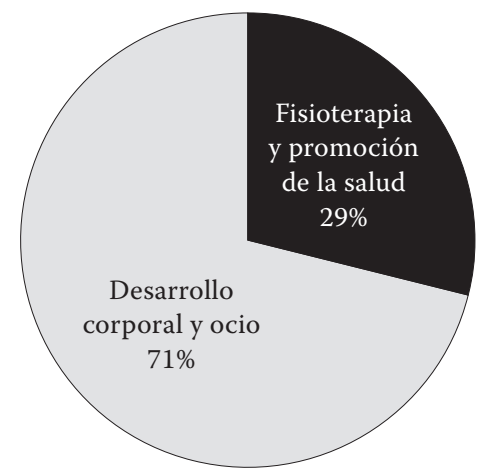

Fuente: elaboración propia

Las dos tendencias restantes, esto es, educación en TIC y evaluación, fueron agrupadas en una única gráfica; ya que entre las dos suman únicamente 4 números y que cada tendencia tiene una sola subdivisión. La primera de ellas, con la subcategoría TIC como mediadoras en el aprendizaje, cuenta con 3 números (75\%). Por su parte, la tendencia temática evaluación, con la subcategoría evaluación y calidad, cuenta con un número (25\%). La distribución en porcentajes se muestra a continuación:

Grafica 7. Educación en TIC y evaluación

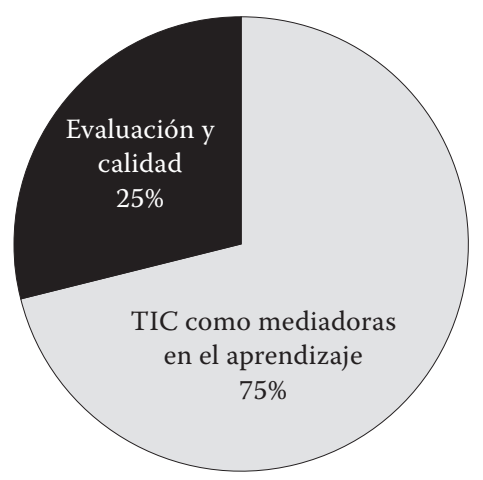

Fuente: elaboración propia 


\section{Discusión de los resultados y conclusiones}

Como lo ponen de manifiesto los resultados obtenidos, la revisión de los últimos 5 números de los principales índices de revistas colombianas especializadas en educación permite identificar qué tendencias temáticas e investigativas han sido las protagonistas en la investigación en educación. Se evidencia una preferencia por dirigir las investigaciones hacia lo denominado practicas pedagógicas, en donde la reflexión por el quehacer docente, la pedagogía y la educación universitaria se vuelven preponderantes. Ospina y Murcia (2012) se refieren al desarrollo de esta tendencia en investigación, en el ámbito nacional, del siguiente modo:

Esta discusión empieza a visibilizarse en la década del $80[\ldots]$ y va tomando mayor fuerza en las últimas dos décadas con la emergencia y desarrollo de fenómenos de carácter nacional como el Movimiento Pedagógico, la superación del enfoque de la tecnología educativa, la reforma de la legislación educativa [...], lo cual se ve hoy reflejado en la producción de conocimiento significativo acerca de las categorías que configuran el gran campo de la pedagogía (Murcia y Ospina, 2012).

A pesar de lo que se pone de manifiesto con lo investigado por Murcia y Ospina, y con los hallazgos hasta ahora expuestos, la investigación en educación, cuyo énfasis se encuentre directamente dirigido a la pedagogía como elemento transversal, no ha sido un elemento crucial en los resultados evidenciados en trabajos de este mismo tipo; tales son los casos de las investigaciones de Murcia y Guacaneme (2012), Ramírez y Gallardo (2012) y Murcia, Murcia y Urbina (2011).

Volviendo a los resultados obtenidos, se puede evidenciar un notorio interés por investigar en áreas que competen al compromiso social, político, ético y cultural; como se evidenció en la tendencia ética y ciudadanía, que cuenta con el segundo porcentaje más alto, conforme a lo analizado.
Por otro lado, los resultados advierten a la comunidad académica, principalmente al conjunto de investigadores de las ciencias de la educación, sobre la falta de investigación concerniente a áreas netamente disciplinares; es decir, investigaciones que centren sus estudios en elementos que sobrepasen los análisis meramente pedagógicos. Los resultados demuestran que el $63 \%$ de las investigaciones (esto es, las tendencias prácticas pedagógicas, pedagogía, aprendizaje y desarrollo y evaluación) se encuentran directamente dirigidas a estudios concernientes a la pedagogía, lo que permite establecer que hay una falta de profundización en las materias, áreas disciplinares. Si bien es cierto que la subcategoría lenguaje y comunicación envuelve la mayor cantidad de investigaciones particulares, es notoria la falta de énfasis en investigaciones ajenas a la pedagogía.

Del mismo modo, y a manera de llamado para las instituciones universitarias, se hace necesario enfatizar en elementos que busquen suplir una de las carencias que existen hoy en día en la educación superior: la mejora de los procesos investigativos. Si bien no se alude a una educación basada en la investigación, sí se hace un llamado por una educación que se encuentre enmarcada en un proceso investigativo de carácter transversal.

De otro lado, si bien para la investigación el idioma no jugó un papel importante en la clasificación de los índices de las revistas en las tendencias temáticas, se destaca el énfasis de cinco revistas por tratar de hacer de sus publicaciones un elemento investigativo con un carácter más global: esto se debe a que únicamente aceptan artículos que se encuentren escritos en inglés, o, en su defecto, en una lengua distinta del español. Si bien esto puede tener repercusiones negativas a nivel nacional -en un país con una población mínima de hablantes bilingües-, desempeña un papel relevante a nivel internacional; sobre 
todo en un mundo académico con un carácter cada vez más global (y unificador) y que se apoya en un idioma particular que unifique. Tal y como lo explica Price (1976): "A Journal which has been frequently consulted for some purpose is more likely to be turned to again than one of previously infrequent use" [Una revista científica que sea frecuentemente consultada por algún motivo, será citada, con mayor probabilidad, que una poco usada $]^{2}$. Y si este medio por el que se citan los artículos es un idioma hoy por hoy institucionalizado (el inglés), habrá una mayor acogida de las investigaciones a nivel mundial.
Finalmente, vale decir que de las 164 revistas indexadas por Colciencias en categorías A1, A2 y B según el último escalafón presentado (Colciencias 2014), solo el 10,3\% se encuentra conformado por aquellas que se enfocan en la investigación educativa; de las cuales, únicamente una se encuentra clasificada en Scimago (un ranking de carácter mundial y, por consiguiente, con mayor impacto investigativo). Esto se convierte en un llamado, tanto a instituciones educativas como a entidades responsables de regular la educación en el país, por hacer de la educación un elemento angular en la investigación nacional.

\section{Referencias}

Colciencias (2015). Listado de Revistas Indexadas II, Actualización Publindex 2014. Recuperado de http://publindex.colciencias.gov.co:8084/publi ndex/EnIbnPublindex/resultados.do

Colciencias (2016). Política para mejorar la calidad de las publicaciones científicas nacionales. Recuperado de http://www.colciencias.gov.co/ sites/default/files/upload/paginas/politicapublindex-colciencias.pdf

Couture, C. (2001). Education and Research in Archival Science: General Tendencies. Archival Science, 1, 157-182. doi: 10.1007/BF02435646

González, H. (2011). Formación investigativa para la educación superior desde una perspectiva pedagógica. Revista cientifica, 14, 72-78. Recuperado de http://revistas.udistrital.edu.co/ ojs/index.php/revcie/article/view/3702

Gu, X. \& Blackmore, K. L. (2016). Recent trends in academic journal growth. Scientometrics, 108, 693-716. doi: 10.1007/s11192-016-1985-3

Hernández, R., Fernández, C. y Baptista, M. (2010). Metodología de la investigación. México D. F.: McGraw-Hill.

Lobato, A. (2010). Representaciones sociales de los docentes sobre la investigación en las facultades de educación: antecedentes, tendencias y ausencias. Revista Educación y Desarrollo Social, 2, 130-142.

2 La traducción es propia.
Ministerio de Educación Nacional (2014). Lineamientos de Calidad para las Licenciaturas en Educación (Programas de Formación Inicial de Maestros). Bogotá D. C. Recuperado de http://www.mineducacion.gov.co/cvn/1665/ articles-340962_recurso_1.pdf

Murcia, N. y Guacaneme, N. (2011). Educación y pedagogía, desde las tendencias en las regiones investigativas en la Universidad Tecnológica de Pereira. Revista Latinoamericana de Estudios Educativos (Colombia), 7(1), 123-140.

Murcia, N., Murcia, N. y Urbina, E. (2011). Tendencias Educativas en educación y pedagogía: estudio de las regiones investigativas de la maestría en educación de la UIS. Revista Latinoamericana de Estudios Educativos (Colombia), 7(1), 81-98. Recuperado de http:// www.redalyc.org/pdf/1341/134125447005.pdf

Nussbaum, M. (2010). Not for Profit: Why Democracy Needs the Humanities (pp. 1-12). New Jersey: Princeton University Press.

Nussbaum, M. (2015). Discurso de aceptación del Doctor Honoris Causa concebido por la Universidad de Antioquia. Recuperado de: http:// www.parqueexplora.org/visitenos/noticias/ discurso-de-martha-nussbaum-al-recibir-eldoctorado-honoris-causa-en-udea/

Ospina, H. y Murcia, N. (Eds.). (2012). Regiones investigativas en educación y pedagogía en Colombia: construcción de un mapa de la 
actividad investigativa de tesis de maestrías y doctorados en el periodo 2000-2010. Manizales: CINDE-Universidad de Manizales.

Price, D. D. S. (1976). A General Theory of Bibliometric and Other Cumulative Advantage Processes. Journal of the American Society for Information Science, 27, 292-306. doi: 10.1002/ asi.4630270505

Quintero, M. y Ruiz A. (2004). ¿Qué significa investigar en educación? (pp. 11-66). Bogotá D. C.: Universidad Distrital Francisco José de Caldas.
Ramírez, C. y Gallardo, B. (2012). Focos de comprensión: perspectivas teóricas. En Ospina, H y Murcia, N. (Eds.). (2012). Regiones investigativas en educación y pedagogía en Colombia: construcción de un mapa de la actividad investigativa de tesis de maestrías y doctorados en el periodo 2000-2010. Manizales: CINDEUniversidad de Manizales.

SCImago (2014). SJR-SCImago Journal \& Country Rank. Recuperado el 27 de febrero de 2016 desde http://www.scimagojr.com

\section{Anexos}

Anexo 1. Información sintetizada de las revistas de educación colombianas incluidas en las categorías $\mathrm{A} 1, \mathrm{~A} 2$, y $\mathrm{B} 1$ de Colciencias.

\begin{tabular}{|c|c|c|c|c|c|c|c|c|}
\hline $\mathbf{N}$. & Nombre de la revista & $\begin{array}{l}\text { Institución a la } \\
\text { que se encuen- } \\
\text { tra asociada }\end{array}$ & $\begin{array}{c}\text { Año de } \\
\text { iniciación } \\
\text { (Latindex) }\end{array}$ & $\begin{array}{c}\text { Año de } \\
\text { indexación } \\
\text { (Publindex) }\end{array}$ & $\begin{array}{c}\text { Ciudad } \\
\text { de } \\
\text { sede } \\
\end{array}$ & $\begin{array}{c}\text { Adscrita a } \\
\text { facultad de } \\
\text { educación }\end{array}$ & $\begin{array}{c}\text { Categoría } \\
\text { Publindex } \\
(2014) \\
\end{array}$ & $\begin{array}{c}\text { Ranking } \\
\text { Scimago } \\
(2014) \\
\end{array}$ \\
\hline 1 & "Profile & UNAL & 2000 & 2006 & Bogotá & No & A1 & No aplica \\
\hline 2 & $\begin{array}{l}\text { "Colombian Applied } \\
\text { linguistics Journal }\end{array}$ & UD & 1999 & 2010 & Bogotá & Sí & A2 & No aplica \\
\hline 3 & Educación y educadores & UNISABANA & 1998 & 2005 & Chía & Sí & A2 & No aplica \\
\hline 4 & Praxis y saber & UPTC & 2009 & 2012 & Tunja & Sí & $\mathrm{A} 2$ & No aplica \\
\hline 5 & $\begin{array}{l}\text { Revista colombiana de } \\
\text { educación }\end{array}$ & UPN & 1979 & 2004 & Bogotá & Sí & A2 & No aplica \\
\hline 6 & $\begin{array}{l}\text { "Revista Historia } \\
\text { de la Educación } \\
\text { Latinoamericana }\end{array}$ & UPTC & 1998 & 2004 & Tunja & Sí & A2 & No aplica \\
\hline 7 & Sophia: educación & UGC & 2005 & 2008 & Armenia & No & A2 & No aplica \\
\hline 8 & "Aletheia & CINDE & 2008 & 2012 & Bogotá & Sí & $\mathrm{B}$ & No aplica \\
\hline 9 & $\begin{array}{l}\text { "Educación física y } \\
\text { deporte }\end{array}$ & UdeA & 1979 & 2010 & Medellín & Sí & B & No aplica \\
\hline 10 & $\begin{array}{l}\text { "GiST Education and } \\
\text { learning research journal }\end{array}$ & ÚNICA & 2002 & 2012 & Bogotá & Sí & B & No aplica \\
\hline 11 & "HOW & ASOCOPI & 1971 & 2009 & Bogotá & No & B & No aplica \\
\hline 12 & $\begin{array}{l}\text { Revista Latinoamericana } \\
\text { de Estudios Educativos }\end{array}$ & UdeC & 2006 & 2010 & Manizales & Sí & B & No aplica \\
\hline 13 & "Lúdica Pedagógica & UPN & 1990 & 2011 & Bogotá & Sí & B & No aplica \\
\hline 14 & Magis & PUJ & 2008 & 2009 & Bogotá & Sí & B & Q4 \\
\hline 15 & Nodos y nudos & UPN & 2000 & 2005 & Bogotá & Sí & B & No aplica \\
\hline 16 & Pedagogía y saberes & UPN & 1990 & 2004 & Bogotá & Sí & $B$ & No aplica \\
\hline 17 & Zona Próxima & Uninorte & 2000 & 2012 & Barranquilla & Sí & B & No aplica \\
\hline 18 & $\begin{array}{l}\text { Journal of Science } \\
\text { Education }\end{array}$ & Varios & 2000 & No aplica & Bogotá & No & No aplica & Q4 \\
\hline
\end{tabular}

* Revista especializada en un área particular de la educación y/o la pedagogía (por ejemplo, educación deportiva, enseñanza del inglés, etc.). 